\title{
Use of discriminant analysis on NIRS to detect meat-and-bone meal content in ruminant concentrates $^{*}$
}

\author{
Z. Yang ${ }^{1,2}$, L. Han ${ }^{1,2,4}$, Q. Li ${ }^{1,2}$ and X. Fan ${ }^{3}$ \\ ${ }^{1}$ College of Engineering, China Agricultural University \\ Beijing 100083, P.R. China \\ ${ }^{2}$ Key Laboratory of Modern Precision Agriculture System Integration, \\ Ministry of Education, Beijing 100083, P.R. China \\ ${ }^{3}$ Institute of Quality and Standards for Agri-Product, Chinese Academy \\ of Agricultural Sciences, Beijing 100081, P.R. China
}

\begin{abstract}
A purpose of this study was to demonstrate the feasibility of using near infrared reflectance spectroscopy (NIRS) to identify MBM (meat-and-bone meal) in the ruminant concentrates. A partial least squares discriminant analysis equation was developed with 235 samples and validated with 59 samples. A calibration model was developed based on spectra region from 1100 to $2498 \mathrm{~nm}$ with mathematic pretreatment 2,4,4,1 and with scatter correction SNVDT (the standard normal variate-detrending). For external validation, there was the accurately discriminant rate of $100 \%$. The results indicated that NIRS could provide a rapidly method for detecting the adulteration of ruminant concentrates with MBM.
\end{abstract}

KEY WORDS: NIRS, discriminant analysis, MBM, ruminant concentrates, adulteration

\section{INTRODUCTION}

Feed contaminated with meat-and-bone meal (MBM) is commonly accepted as the main transmission carrier of bovine spongiform encephalopathy (BSE). Consumers and the feed industry need to have appropriate methods available to detect such a contamination or adulteration.

\footnotetext{
* Supported by National Natural Science Foundation of PRC, Contract 30571074 and Project in the National Science \& Technology Pillar Program, Contract 2006BAD12B03-03

${ }^{4}$ Corresponding author: e-mail: Hanlj@cau.edu.cn; yangzengling@163.com
} 
Near infrared reflectance spectroscopy (NIRS) is probably the most rapid method for testing feed in terms of speed of reporting, timeliness and convenience, and providing an instant response in detecting contaminated specimens. NIRS has been already applied widely to the routine analysis in the feed industry (PérezMarín et al., 2004a) and is the most likely technique to be used to protect the feed from contamination with MBM.

Some authors have reported the calibration equations to quantify the percentage of MBM in the compound feeds and to classify samples according to the presence/ absence of MBM (Fernández et al., 2002; Dolores et al., 2004; Murray et al., 2004; Pérez-Marín et al., 2004b; Termes et al., 2004). Furthermore, EU has instigated a research program called STRATFEED (Contract G6RD-CT2000-00414) that explored new strategies for the detection of mammalian tissues in feedstuffs (Murray et al., 2005).

Adulteration of ruminant concentrates with MBM is the most riskful for spread of BSE and this adulteration is severely forbidden in People's Republic of China. A purpose of this study was to demonstrate the feasibility of NIRS method to identify MBM in the ruminant concentrates.

\section{MATERIAL AND METHODS}

\section{Calibration set and validation set}

Commercial ruminant concentrates were collected from around China. The MBM samples were acquired mainly from protein feed mills of Hebei Province but also from other provinces. These MBM samples included meat-and-bone meal (cattle, sheep, pig and poultry) and meat meal (cattle, sheep, pig and poultry). The samples were in dried, ground form and contamination was achieved by combining the required mass ratios and then mixing thoroughly.

Calibration set consisted of 235 samples including 100 pure ruminant concentrates and 135 adulteration samples. The 135 adulteration samples were prepared in laboratory used 135 ruminant concentrates and 45 MBM samples. In detail, adulterated samples were spiked with varying percentages $(0.5-35 \%$, as feed) of 45 MBM samples according to a factorial design of randomized blocks (Garrido-Varo et al., 2005). Three samples were prepared at each concentration. Each ruminant concentrates was used once only. Each MBM was used three times randomly.

The external validation set, made up of 59 samples including 14 pure samples and 45 adulterated samples which were spiked with varying percentages $(0.5-35 \%)$ of 45 MBM samples randomly. One sample was prepared at each concentration. Each ruminant concentrates and MBM were used once only. 


\section{NIRS analysis}

The scanning monochromator NIRSystems ${ }^{\mathrm{TM}} 6500$ visible-NIR (FOSS, UK), equipped with a transport module, was use to measure reflectance spectra as $\log (1 / \mathrm{R})$ from 400 to $2498 \mathrm{~nm}$ at $2 \mathrm{~nm}$ intervals, thus covering the visible spectrum as well as the NIR regions. The spectra were recorded with the WinISI II software (Infrasoft International, Port Matilda, PA, USA). Considering packing density, every sample was scanned three times by three different people. Three spectra of each sample were all used to develop the calibration equation. In scanning process, samples were scanned in random sequence so that no instrumental bias was produced.

\section{NIRS data treatment}

In order to reduce baseline offset arising from particle size and packing density, the data were treated using mathematical treatments and scatter correction treatment. The effect of three different mathematic pretreatments and seven scatter correction algorithms were tested. There were 21 permutations which PLS discriminant equations were developed.

The calibration method was the Partial Least Squares (PLS) discriminant analysis. An uncertainty factor of 2.5 was chosen by default. Cross validation is conducted to test the accuracy of the discrimination and help determine how many factors to include in the final equation. Calibration statistics calculated include the number of uncertain samples, the standard error of calibration (SEC), the coefficient of multi-determination in calibration $\left(\mathrm{R}^{2}\right)$, the standard error of crossvalidation (SECV) and the coefficient of determination in cross-validation $\left(\mathrm{R}_{\mathrm{cv}}{ }^{2}\right)$. The statistic used to select the best equation was SECV.The external validation set was used to validate the capacity of calibration equation.

\section{RESULTS AND DISCUSSION}

Near infrared spectroscopy uses the global molecular fingerprint of the samples to detect the presence of MBM. In order to investigate the difference between ruminant concentrates and MBM from spectrum characteristic, $45 \mathrm{MBM}$ samples and 135 ruminant concentrates were used to create principal component scores, and then they were used to calculate Global H (GH) distances. This was done using the mean of 135 ruminant concentrate samples as the centre $(\mathrm{GH}=0)$. $\mathrm{GH}$ distances of 135 ruminant concentrate samples were less than 3.0. GH distances of $45 \mathrm{MBM}$ samples were greater than 3.0. This difference may explain the basis for discrimination. 
The calibration results are given in Table 1 . The effect of mathematic treatments is greater than scatter corrections. Using first derivative and second derivative to transform the data was helpful for calibration equation. The effect of scatter corrections on calibration equation was variable with different samples. However, the effect was not significant. In this study, the mathematic treatment 2,4,4,1 with SNVDT scatter correction was chosen to be the best spectra pretreatment method based on SECV.

Table 1. PLS discriminant analysis for MBM adulteration of ruminant concentrates $(n=705)$

\begin{tabular}{|c|c|c|c|c|c|c|c|c|}
\hline$\lambda$ & $\begin{array}{l}\text { Mathematic } \\
\text { pretreatments }\end{array}$ & $\begin{array}{c}\text { Scatter } \\
\text { corrections }\end{array}$ & $\begin{array}{l}\text { PLS } \\
\text { terms }\end{array}$ & Uncertainty & $\mathrm{R}^{2}$ & SEC & $\mathrm{R}_{\mathrm{cv}}{ }^{2}$ & SECV \\
\hline \multirow{7}{*}{260} & \multirow{7}{*}{$0,0,1,1$} & None & 29 & 343 & 0.84 & 0.1983 & 0.82 & 0.2118 \\
\hline & & SNVDT & 30 & 321 & 0.83 & 0.2022 & 0.81 & 0.2179 \\
\hline & & SNV & 29 & 374 & 0.79 & 0.2257 & 0.76 & 0.2409 \\
\hline & & DT & 30 & 353 & 0.81 & 0.2155 & 0.78 & 0.2316 \\
\hline & & Std MSC & 29 & 340 & 0.82 & 0.2105 & 0.79 & 0.2240 \\
\hline & & Wtd MSC & 30 & 307 & 0.84 & 0.1961 & 0.82 & 0.2108 \\
\hline & & Inv MSC & 29 & 323 & 0.84 & 0.1994 & 0.81 & 0.2147 \\
\hline \multirow{7}{*}{259} & \multirow{7}{*}{$1,4,4,1$} & None & 29 & 141 & 0.93 & 0.1314 & 0.91 & 0.1515 \\
\hline & & SNVDT & 28 & 130 & 0.93 & 0.1306 & 0.91 & 0.1501 \\
\hline & & SNV & 30 & 116 & 0.94 & 0.1242 & 0.91 & 0.1447 \\
\hline & & DT & 30 & 151 & 0.93 & 0.1334 & 0.90 & 0.1555 \\
\hline & & Std MSC & 30 & 118 & 0.94 & 0.1241 & 0.91 & 0.1448 \\
\hline & & Wtd MSC & 29 & 136 & 0.93 & 0.1291 & 0.91 & 0.1491 \\
\hline & & Inv MSC & 30 & 111 & 0.94 & 0.1248 & 0.92 & 0.1430 \\
\hline \multirow{7}{*}{256} & \multirow{7}{*}{$2,4,4,1$} & None & 20 & 46 & 0.94 & 0.1184 & 0.91 & 0.1467 \\
\hline & & SNVDT & 28 & 34 & 0.96 & 0.1028 & 0.93 & 0.1332 \\
\hline & & SNV & 28 & 34 & 0.96 & 0.1030 & 0.93 & 0.1334 \\
\hline & & DT & 20 & 45 & 0.94 & 0.1183 & 0.91 & 0.1463 \\
\hline & & Std MSC & 28 & 35 & 0.96 & 0.1030 & 0.93 & 0.1334 \\
\hline & & Wtd MSC & 27 & 33 & 0.96 & 0.1032 & 0.93 & 0.1343 \\
\hline & & Inv MSC & 28 & 34 & 0.96 & 0.1031 & 0.93 & 0.1333 \\
\hline
\end{tabular}

To assess the relative contributions of the visible $(400-1098 \mathrm{~nm})$ and the NIR (1100-2498 nm) taken alone and together, calibration set was used to develop calibration in three regions (Vis, NIR, and Vis+NIR) (Table 2). The visible region alone performed the bad result and had no contribution to the combined performance. The NIR region did produce a very good calibration result which was better than combination of visible and NIR region. NIR region (1100-2498 $\mathrm{nm})$ was as the final spectra region according to minimum SECV. 
Table 2. Comparison of visible and NIR regions in discrimination $(n=705)$

\begin{tabular}{llccccccc}
\hline $\begin{array}{l}\text { Pre- } \\
\text { treatment }\end{array}$ & Range & $\lambda$ & $\begin{array}{c}\text { PLS } \\
\text { terms }\end{array}$ & Uncertainty & $\mathrm{R}^{2}$ & SEC & $\mathrm{R}_{\mathrm{cv}}{ }^{2}$ & SECV \\
\hline \multirow{2}{*}{$2,4,4,1$} & Vis & 84 & 10 & 320 & 0.74 & 0.2515 & 0.69 & 0.2744 \\
SNVDT & NIR & 172 & 25 & 47 & 0.94 & 0.1162 & 0.92 & 0.1393 \\
& Vis+NIR & 256 & 20 & 46 & 0.94 & 0.1184 & 0.91 & 0.1467 \\
\hline
\end{tabular}

The calibration equation was validated by external validation set (Figure 1). MBM-free ruminant concentrate were grouped together in one cluster around a score of 2.0, while samples containing different levels of MBM cluster around 1.0 with a breakpoint score of 1.5. The sample scores between two broken lines $[1.5 \pm$ Uncertain Factor $(2.5) * \mathrm{SECV} / 2]$ were uncertainty samples. It is uncertain which group these samples should belong to. For validation result (Figure 1), there was no uncertain sample. The accurately dicriminant rate was $100 \%$. The validation results indicated that NIRS method can rapidly discriminate the MBM content in ruminant concentrates.

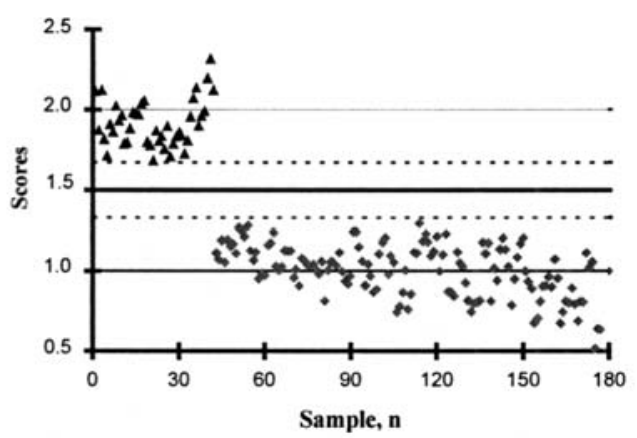

Figure 1. The results of validation for calibration on NIR region

\section{CONCLUSIONS}

The results of the discriminant analysis indicated that NIRS could provide a rapidly screening method for detecting the adulteration of ruminant concentrates with meat-and-bone meal.

\section{ACKNOWLEDGEMENTS}

Special thanks goes to Dr. V. Baeten (Walloon Agricultural Research Centre, Gembloux, Belgium) for his helpfulness and expert suggestions. The authors also gratefully acknowledge the assistance of Zhao Wushan (FOSS, China). 


\section{REFERENCES}

Dolores C., Pérez-Marín D., Garrido-Varo A., Guerrero-Ginel J.E., Gómez-Cabrera A., 2004. Nearinfrared reflectance spectroscopy (NIRS) for the mandatory labeling of compound feedingstuffs: chemical composition and open-declaration. Anim. Feed Sci. Tech. 116, 333-349

Fernández M., Martínez A., Modroños., De la Roza B., 2002. Near infrared reflectance spectroscopy as a tool to predict qualitative and quantitative meat and bone meal presence in compound feed. In: A.M.C. Davies, R.K. Cho (Editors). Proceedings of the 10th International Conference. Near Infrared Spectroscopy. NIR Publications. Chichester, West Sussex (UK), pp. 307-312

Garrido-Varo A., Pérez-Marín D., Guerrero J.E., Gómez-Cabrera A., Von Holst C., Murray I., Van Raamdonk L.W.D., Zegers J., 2005. Construction of the Stratfeed sample bank and preparation of sample sets (WP2). In: Stratfeed. Strategies and Methods to Detect and Quantify Mammalian Tissues in Feedingstuffs. Office for Official Publications of the European Communities

Murray I., Garrido-Varo A., Pérez-Marín D., Guerrero J.E., Baeten V., Dardenne P., Termes S., Zegers J., Frankhuizen R., 2005. Macroscopic near-infrared reflectance spectroscopy (WP5). In: Stratfeed. Strategies and Methods to Detect and Quantify Mammalian Tissues in Feedingstuffs. Office for Official Publications of the European Communities

Murray I., Pérez-Marín D., Garrido-Varo A., Guerrero J.E., Puigdomènech A., Dardenne P., Baeten V., Zegers J., 2004. STRATFEED near infrared instrument network for detecting animal tissues in feedingstuffs. In: A.M.C. Davies, A. Garrido (Editors). Proceedings of the 11th International Conference. Near Infrared Spectroscopy. NIR Publications. Chichester, West Sussex (UK), pp. 291-294

Pérez-Marín D., Garrido-Varo A., Guerrero J.E., Gómez-Cabrera A., 2004a. Near-infrared reflectance spectroscopy (NIRS) for the mandatory labeling of compound feedingstuffs: chemical composition and open-declaration. Anim. Feed Sci. Tech. 116, 333-349

Pérez-Marín D., Garrido-Varo A., Guerrero J.E., Murray I., Puigdomènech A., Dardenne P., Baeten V., Zegers J., 2004b. Detection and quantification of mammalian meat \& bone meal in compound feedingstuffs by NIR spectroscopy. In: A.M.C. Davies, A. Garrido (Editors). Proceedings of the 11 th International Conference. Near Infrared Spectroscopy. NIR Publications. Chichester, West Sussex (UK), pp. 667-672

Termes S., Tauler R., Puigdomènech A., 2004. Preliminary results in the determination of meat and bone meal in feedingstuffs. In: A.M.C., Davies, A. Garrido (Editors). Near Infrared Spectroscopy. Proceedings of the 11th International Conference. NIR Publications. Chichester, West Sussex (UK), pp. 659-662 